\title{
Brain involvement in Duchenne muscular dystrophy: a role for dystrophin isoform Dp71 in cell migration and proliferation
}

\author{
Amanda Ash ${ }^{1}$, Leah Booth-Wynne ${ }^{1}$ and Karen Anthony ${ }^{1^{*}}$ \\ ${ }^{1}$ Faculty of Health and Society, University of Northampton, Northampton, UK. \\ *Presenting author
}

Intelligence of individuals with Duchenne muscular dystrophy (DMD) is lower than the general population. Cognitive impairment, epilepsy, autism and attention deficit hyperactivity disorder are prevalent. Mounting evidence links these symptoms to the loss of dystrophin in the brain; yet central nervous system involvement in DMD has been largely ignored. The most predominant dystrophin isoform in the brain is the smaller Dp71 isoform expressed in neurons and glia. Distal DMD mutations affecting Dp71 expression are linked to cognitive impairment. The function(s) of Dp71 and how its absence can lead to cognitive and behavioural symptoms are not well understood. We are modelling the loss of Dp71 both endogenously in DMD patient-derived fibroblasts (which, in controls, naturally express Dp71) and with siRNA in neuronal and glial cell lines. Four patient fibroblast cell lines, each harbouring a different $D M D$ gene mutation that naturally results in the loss of $D p 71$ are being studied. Accompanying clinical data confirms these patients display cognitive and/or behavioural symptoms, which is severe for two individuals. Our preliminary findings highlight a role for Dp71 in cell migration and proliferation; alterations in these processes are associated with psychiatric disorders prevalent in DMD such as autism. We will present would healing and proliferation assay data exploring these role(s) and discuss potential molecular mechanisms behind the cognitive and behavioural symptoms observed in DMD. The high risk of neuropsychiatric syndromes in Duchene warrants early intervention to achieve the best possible quality of life; our findings have significant potential to inform ongoing drug development in this area. 